\title{
Prospection archéologique et échantillonnage : un divorce paradoxal
}

\author{
Archaeological survey and sampling: a paradoxical divorce
}

\author{
François Djindjian ${ }^{1}$ \\ ${ }^{1}$ CNRS UMR 7041 Arscan, francois.djindjian@mae.u-paris10.fr
}

\begin{abstract}
RÉSUMÉ. Le présent article traite de la question de l'échantillonnage dans la prospection archéologique, et particulièrement dans l'archéologie préventive. Après avoir rappelé les origines de l'échantillonnage en archéologie depuis les années 1960, et les travaux des archéologues français dans ce domaine dans les années 1970, la méthode de prospection employée par l'INRAP, basée sur le sondage par transects à la pelle mécanique, est étudiée et son efficacité critiquée. Des améliorations sont fournies. Puis, il est proposé de distinguer en archéologie préventive la prospection archéologique à grande échelle qui s'effectue en amont avec des techniques non destructives, de la prospection pour diagnostic qui est réalisée généralement après la phase d'acquisition du foncier. Il est démontré alors que les deux prospections sont indispensables et complémentaires, la première permettant d'enrichir l'application de la seconde. Une méthodologie générale permettant de spécifier un plan de prospection est alors définie utilisant au mieux les nombreuses techniques de prospections disponibles. Certaines de ces techniques ont connu récemment des améliorations importantes en termes de fiabilité et de productivité qui augmentent profondément l'efficacité d'un plan de prospection. Enfin, la composante politique de la méthode actuelle de I'INRAP est évoquée, et il est proposé de bien séparer le traitement scientifique du sujet qui concerne la définition de la méthode de son traitement social qui concerne en France l'organisation métier de sa mise en œuvre.

ABSTRACT. This article deals with the issue of sampling in archaeological survey, and particularly in preventive archaeology. After recalling the origins of sampling in archaeology since the 1960s, and the work of French archaeologists in this area in the 1970s, the method of prospecting used by INRAP, based on the survey by transects dug by excavator, is studied and its effectiveness criticized. Improvements are given. Then, it is proposed to distinguish for preventive archaeology between the archaeological prospecting on a large scale that occurs upstream with nondestructive techniques, and prospecting for diagnosis that is performed generally after the land acquisition phase. It is then shown that the two surveys are essential and complementary, the first allowing to enrich the application of the second. A general methodology for specifying a prospecting plan is then set using the many techniques of surveys available at best. Some of these techniques have recently experienced significant improvements in terms of reliability and productivity which increase deeply the effectiveness of a prospecting plan. Finally, the political component of the current method of the INRAP is mentioned, and it is proposed to separate the scientific treatment of the subject that concerns the definition of the method from its social treatment that includes the business organization of its implementation in France.

MOTS-CLÉS. archéologie, prospection, échantillonnage, archéologie préventive.

KEYWORDS. archaeology, survey, sampling, rescue archaeology.
\end{abstract}

\section{Introduction}

C'est au début des années 1960 que les archéologues ont pris conscience des liens étroits entre les données issues des fouilles et des prospections archéologiques et de la notion d'échantillonnage probabiliste (Vescelius, 1960). En effet, les fouilles n'étant qu'exceptionnellement exhaustives et les prospections dépendant à la fois de l'approche plus ou moins systématique de la prospection, de la capacité de détecter les sites et de la survivance de ces sites liées à des évènements naturels et anthropiques, l'acquisition des données n'est que partielle. Elle n'est donc que le résultat d'un échantillonnage empirique. En conséquence, tout inventaire, tout comptage, toute mesure sur des objets archéologiques ou environnementaux (comme des prélèvements de sédiments ou de pollens par exemple) ne sont que des estimations issues de cet échantillonnage empirique, et dont on ne sait pas estimer le biais a priori (Desabie, 1966, pour les bases mathématiques de l'échantillonnage). Bien plus, l'absence d'un site ou d'un objet pouvant signifier aussi bien son absence définitive que sa non- 
découverte, l'archéologie fonctionne sur une logique ternaire (présence, absence, non-découverte) qui complexifie les argumentations et en fait une des disciplines les plus ingrates du domaine scientifique. Un des enjeux majeurs de la méthode archéologique est donc de calculer en termes statistiques l'erreur de la moyenne et la valeur de l'écart-type de la grandeur considérée, ce que fait un échantillonnage probabiliste.

L'échantillonnage n'est pas né de la recherche académique en archéologie. Il est né de l'archéologie préventive. Les grandes opérations de sauvetage liées aux grands travaux d'aménagement ont conduit les archéologues nord-américains à aborder ces questions d'un point de vue méthodologique, dans les années d'après-guerre, concrétisées par le livre "Sampling in Archaeology» (Mueller, 1975). L'archéologie préventive anglaise, qui a démarré entre temps, les suit: "The role of sampling in contemporary British Archaeology" (Cherry \& al. 1978). Ces recueils d'articles relatent des expérimentations le plus souvent infructueuses mais à ce titre encore plus instructives.

\section{Archéologie et échantillonnage dans l'archéologie française dans les années 1980}

Les premiers essais français d'échantillonnage probabiliste en archéologie datent, à ma connaissance, des travaux de l'auteur de cet article, dans le cadre du séminaire "Informatique et mathématiques appliquées à l'archéologie » des années 1980, à Paris. Un des sujets traités était l'échantillonnage qui a donné lieu en 1988 à un polycopié, distribué mais non édité, dont une partie des résultats a été publié dans le livre « Méthodes pour l'archéologie » (Djindjian, 1991, chapitres 3 et 4).

L'efficacité d'une technique d'échantillonnage ne peut en fait s'évaluer qu'en effectuant des simulations d'échantillonnage sur des données considérées comme exhaustives : pour la prospection régionale, des cartes de sites archéologiques et pour les fouilles archéologiques des distributions spatiales de structures et d'artefacts de larges zones décapées.

Les conclusions des simulations effectuées à cette époque, peuvent être résumées comme suit :

- En prospection régionale, la précision et l'efficacité des plans d'échantillonnage probabiliste sont très faibles sauf à atteindre des taux de l'ordre de $25 \%$,

- En prospection intrasite, seul le plan au troisième degré fournit une efficacité acceptable mais à un taux d'échantillonnage qui ne peut guère descendre sous les $5 \%$.

L'échantillonnage probabiliste est donc une méthode insuffisante pour des raisons inhérentes à la variabilité et à la morphologie des distributions spatiales des sites pour la prospection, ou des structures d'habitat et de leur superficie au sol pour les sites archéologiques. Ceci explique le manque d'intérêt suscité par cette approche à partir des années 1980 .

En particulier, l'échantillonnage probabiliste est particulièrement peu adapté à la découverte de structures archéologiques de faible couverture spatiale. Ainsi, sur l'exemple ci-contre (figure 1), avec un échantillonnage probabiliste systématique non aligné par transects avec un taux de 12,5\%, 3 structures archéologiques sur les 8 présentes ne sont pas détectées (qui peuvent être considérées soit comme des indices de sites archéologiques dans le cas d'une prospection, soit comme des structures d'habitat dans le cas d'une fouille d'un site). 


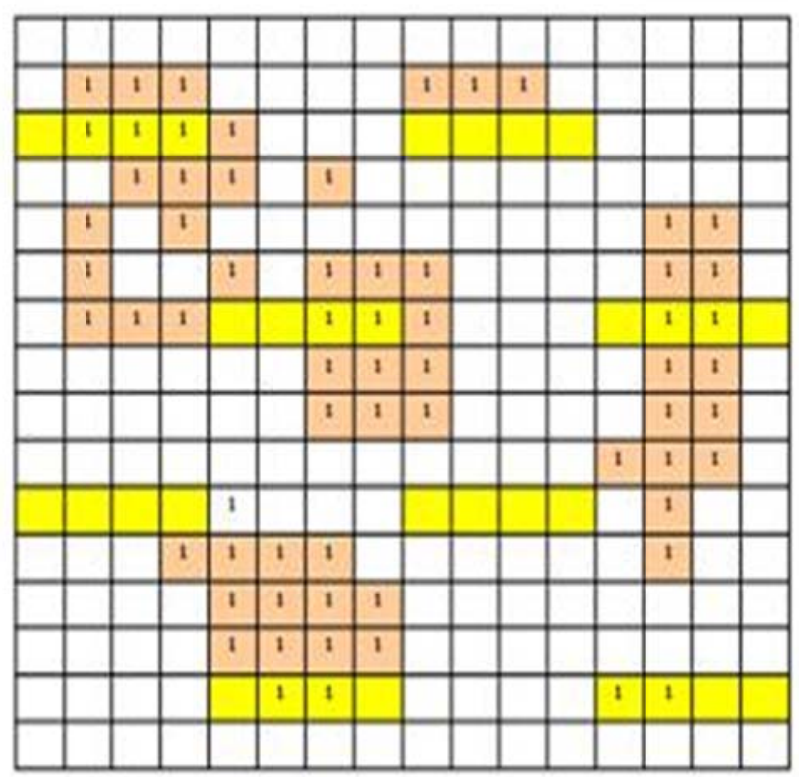

Figure 1. Simulation d'un échantillonnage probabiliste systématique non aligné par transects avec un taux de 12,5\% (3 structures archéologiques sur les 8 présentes ne sont pas détectées)

Cet exemple nous rappelle les questions récurrentes qui se posent dans l'échantillonnage archéologique :

- Quelle taille de transects définir?

- Quel taux d'échantillonnage?

- Quel plan d'échantillonnage?

- Échantillonnage systématique (non aligné),

- Échantillonnage systématique à taux variable à plusieurs degrés,

- «Échantillonnage raisonné» ou plan de sondage non systématique adapté au terrain et aux connaissances archéologiques sur le terrain

En conclusion, l'échantillonnage probabiliste ne peut être considéré que comme la première étape d'une approche archéologique « in terra incognita », quand aucune autre information ne permet de guider un plan de prospection ou une conduite de fouilles. Il doit donc être complété par d'autres méthodes pour améliorer son efficacité.

\section{La prospection dans l'archéologie française}

Sans aborder la question de l'échantillonnage probabiliste, les archéologues français ont pratiqué, dès les années 1970, la prospection et amélioré sa méthodologie :

- Dans le cadre de l'archéologie française à l'étranger, c'est le cas par exemple de la prospection de Jean-Claude Gardin en Bactriane de 1974 à 1978 dans le cadre de la DAFA (Gardin, 1998), qui a permis sur une première superficie de $200 \mathrm{~km}^{2}$ de déceler 374 sites puis sur une superficie plus large de 1500 $\mathrm{km}^{2}$ de déceler 474 sites, de démontrer que les canaux d'irrigation remontent à la période chalcolithique et d'y découvrir un site harappéen (Shortugai). A cette époque, dans cette zone semi-désertique, les études cartographiques et géomorphologiques, les ramassages de surface et une typologie des céramiques (avec sélection et échantillonnage des tessons) ont été les principaux outils de la méthode de prospection. 
- En France, la prospection menée par J. Chapelot, S. Renimel et A. Hesse au début des années 1970 dans le bassin de la Charente, sur la production, l'embarquement et la distribution de la céramique saintongeaise à partir du $\mathrm{XIII}^{\circ}$ siècle, est un bon exemple de prospection intégrant la recherche d'archives, le ramassage de surface, la prospection géophysique et les fouilles subaquatiques (Cartier et al., 1972).

- Il faut également souligner les travaux d'A. Hesse, fondateur en France de la prospection géophysique. Il a également publié des travaux novateurs sur les méthodes de ramassage de surface en terrain semi-désertique et sur le traitement des données spatiales des artefacts collectés, séparant ainsi les sites archéologiques d'époque différente, que l'absence de sédimentation avait superposée (Hesse, 1994). Ces résultats sont toujours d'actualité.

- Un projet significatif, dans le cadre des débuts de l'archéologie de sauvetage, à l'époque de l'Afan, a été mené par les archéologues de la région Centre sur le tracé de l'autoroute A71 de 1979 à 1983 suivi à partir de 1989 des évaluations et des fouilles de sites repérés (Ferdière et Rialland, 1995). La publication de Ferdière et Rialland est particulièrement intéressante car il s'agit d'une des premières expérimentations en vraie grandeur entre archéologues et aménageurs. Les techniques de prospection utilisées ont été la documentation préalable, la prospection à vue, le ramassage de surface, le carottage à la tarière, la prospection aérienne, les prises de vues verticales, la prospection géophysique par thermographie aéroportée. La prospection a permis la découverte de nombreux parcellaires et de 237 sites. Un des aspects les plus intéressants de cette publication est la comparaison faite entre la phase de prospection, la phase d'évaluation et la phase de fouilles sur les sites repérés. La conclusion des auteurs est prémonitoire: "D’un autre point de vue, préconiser la réalisation du recensement des sites archéologiques suffisamment en amont de la définition d'un tracé autoroutier définitif n'est pas seulement apporter une réponse technique et scientifique face à un problème donné. C'est une sorte d'idéologie à défendre (on pourrait même dire une certaine déontologie) qui permettrait peut-être (à un moment où les rapports archéologues/aménageurs sont pour le moins tendus) de corriger certaines attitudes négatives caricaturées à dessein: pour les aménageurs, ne pas avoir la douloureuse impression que les fouilles archéologiques constituent seulement une obligation (voire une fatalité) à laquelle ils ne peuvent se soustraire, et pour les seconds de reconsidérer les choix des interventions (voire l'absence de choix, plus sur la base d'une connaissance scientifique d'ensemble qu'en fonction de la capacité du premier à payer (la bonne aubaine!) des fouilles, certes onéreuses, mais rendues obligatoires sur un tracé imposé et figé. Utopie ou défi ? Discours iconoclaste ou réflexion de bons sens ? » (Ferdière et Rialland, 1995, p. 72).

Ainsi, en conclusions, l'archéologie française a connu, dans les années 1970, une activité de prospection significative menée par les archéologues chercheurs et universitaires, aussi bien dans une approche programmée que dans une approche d'archéologie de sauvetage, qui se concrétiseront notamment par de nombreuses publications, une table ronde à Paris en 1982 (Ferdière et Zadora-Rio, 1986) et une synthèse publiée plus tardivement, indispensable bien qu'inégale (Dabas et al., 1998, réédition 2006).

\section{Prospection archéologique, archéologie préventive et échantillonnage}

L’archéologie préventive française, comme la «Rescue Archaeology» anglo-saxonne, a été confrontée dès les débuts de l'Afan puis, après la création de l'INRAP, à la question de l'échantillonnage dans le cadre des opérations archéologiques sur de grandes superficies (lignes de TGV, autoroutes, canaux, sites industriels et commerciaux).

La méthode, qui devint la bible de l'INRAP, est une prospection systématique non alignée par des transects ouverts à la pelle mécanique (largeur de godet jusqu'à 1,80 m) à un taux d'échantillonnage de 5\% (Blouet V., 1994) : 
«Cette méthode a été développée par le Service régional de l'archéologie de Lorraine qui l'avait testé en 1994, sur une superficie de 5000 ha. Cette méthode peut être considérée comme destructrice car elle utilise des moyens lourds (pelle mécanique), qu'elle évacue la couche de labour et qu'elle détruit parfois des réseaux existants (drainage, chemins etc.). Elle nécessite par ailleurs une remise en état des terrains si elle n'est pas suivie directement par l'aménagement. Elle est fortement dépendante du projet d'aménagement auquel elle est rattachée puisqu'elle nécessite l'acquisition des parcelles foncières et qu'elle ne peut se développer en dehors de l'emprise de l'opération. Elle intervient donc dans une phase relativement avancée du projet, après les études impacts et la déclaration d'utilité publique et généralement, après la phase d'acquisition du foncier » (Robert S., 2003).

Les bonnes pratiques d'échantillonnage préconisent d'utiliser des transects non alignés (autrement dit en quinconce) de longueur de 15 à 20 mètres. Mais la pratique de longs transects parallèles, dont l'efficacité est encore plus faible à taux d'échantillonnage égal, semble s'être aussi développée pour des raisons certes de simplicité d'utilisation des pelles mécaniques mais sans justification méthodologique. En outre, la largeur des godets utilisables, avec l'évolution des technologies depuis les années 2000, peut atteindre 3 mètres, ce qui permet de faire passer le taux d'échantillonnage de 5 à $10 \%$, à nombre de transects constant.

On peut certes regretter que V. Blouet n'ait pas lu ma publication de 1991 où le sujet était traité, car il a conclu à tort qu'un taux d'échantillonnage de 5\% était suffisant. Mais surtout, son approche méthodologique n'était pas argumentée. Seule, la comparaison des coûts l'était, entre les différentes techniques de prospection de l'époque (mais l'automatisation de ces techniques rend cette comparaison aujourd'hui obsolète, cf. infra) et les sondages par transects à la pelle mécanique.

Cette méthode est toujours utilisée aujourd'hui bien que des critiques aient été formulées à son encontre. Une de ces critiques, publiée en 2002, est l'article : «Prospection systématique par sondages à la pelle mécanique : limites liées à la probabilité de découverte de sites archéologiques » (Marmet et al. 2002). L'article calcule la probabilité de découverte d'un site avec un plan d'échantillonnage systématique en quinconce en fonction du taux d'échantillonnage et de la longueur des transects. Il confirme sans surprise que la probabilité avec un taux d'échantillonnage de $10 \%$ sur des transects $2 \mathrm{x}$ $20 \mathrm{~m}^{2}$ est trop faible.

\section{Améliorations méthodologiques}

Dans le livre de 1991 (Djindjian, 1991) comme dans le manuel d'archéologie de 2011 (Djindjian, 2011), nous avions souligné que le sondage à la pelle mécanique comme échantillonnage probabiliste n'était qu'une parmi les nombreuses techniques de prospection à la disposition de l'archéologue. Pour améliorer son efficacité, deux méthodes, qui existaient déjà dans les années 1980, sont utiles : le krigeage et les modèles prédictifs.

Le krigeage, mis au point par l'ingénieur sud-africain D. G. Krige dans les années 1950, est une méthode d'interpolation spatiale utilisée dans la prospection minière mais déjà empiriquement connue à la fin du XIX ${ }^{\circ}$ siècle. Il faut en effet relire Jack London quand il relate dans sa nouvelle « Le Val Tout-en-Or » comment découvrir l'endroit de plus grande richesse d'un filon d'or alluvionnaire (London, 2009, p.744-758). La méthode a été remarquablement généralisée par le français G. Matheron dans les années 1960 (Matheron, 1962, 1971). Le krigeage améliore la prospection si une structure a été détectée par un échantillonnage préalable. La figure 2 fournit les résultats de l'application d'un krigeage sur le plan aléatoire systématique non aligné à $12,5 \%$ de la figure 1 . La comparaison entre la population réelle (à gauche) et la population interpolée par krigeage (à droite) révèle l'efficacité de la méthode. Celle-ci est particulièrement utile dans le cadre de fouilles sur un site archéologique de grande superficie où elle apporte un gain de $50 \%$ dans l'effort de sondage. 


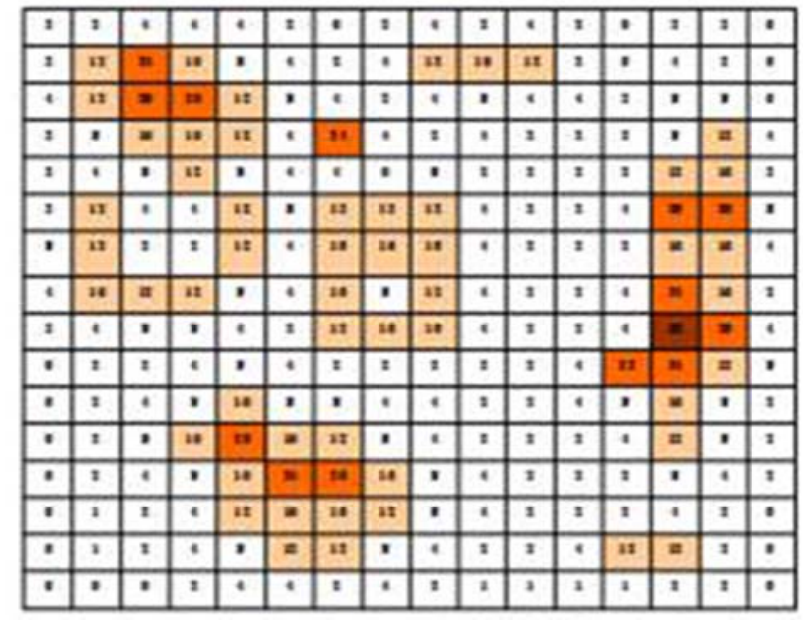

Figure 2.a

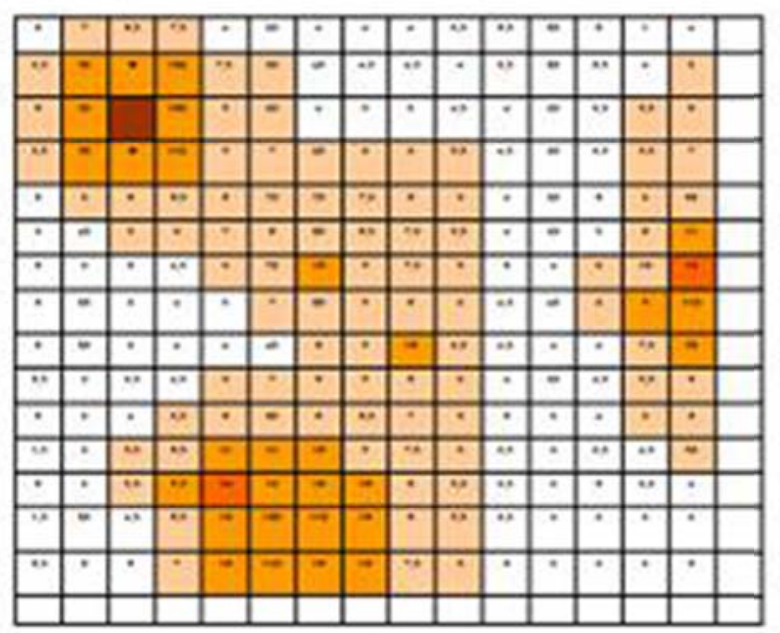

Figure 2.b

Figure 2. Simulation de l'application d'une technique de krigeage sur le plan aléatoire systématique non aligné à $12,5 \%$ de la figure 1 . Figure $2 a$ : relevé exhaustif; Figure $2 b$ : relevé après krigeage sur échantillonnage $12,5 \%$ à comparer avec la figure 1.

Appliqués pour la première fois dans les années 1980 aux Etats-Unis, les modèles prédictifs ont retrouvé un regain d'intérêt dans les années 1995, avec le développement de systèmes d'information géographique, les nombreuses données cartographiques, environnementales et géomorphologiques facilement disponibles et les cartes archéologiques. Un modèle prédictif fournit notamment une carte de probabilité de présence de sites archéologiques dans la région considérée. C'est le cas, par exemple (figure 3), des sites paléo-indiens des régions de la Forêt Nipissim et du Lac Burrows dans l'Ontario au Canada (d'après L. dalla Bona, in « Wescott et Brandon, 2000 », fig. 5.4 p.85).

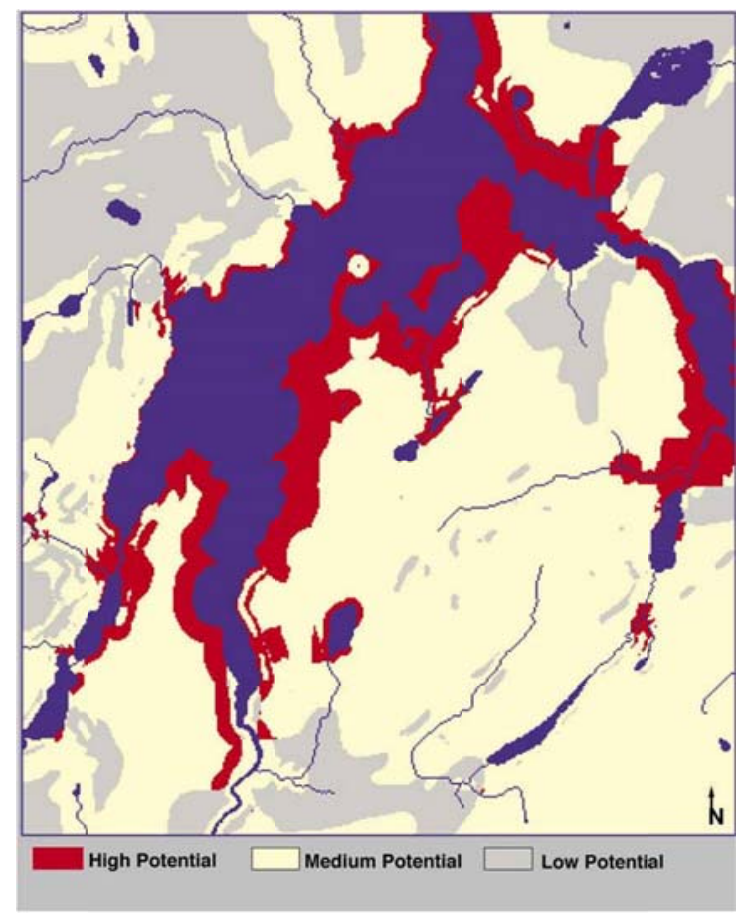

Figure 3. Réalisation d'un modèle prédictif pour la détection des sites paléo-indiens des régions de la Forêt Nipissim et du Lac Burrows dans l'Ontario au Canada (d'après L. dalla Bona, in « Wescott et Brandon, 2000 », fig. 5.4 p.85). 
Il faut faire remarquer le peu d'intérêt pour l'application de modèles prédictifs dans l'archéologie française. Parmi les pays qui se sont le plus investis dans ce thème de recherche, nous pouvons citer notamment les Pays-Bas (Van Leusen et Kamermans, 2005). Ce sont des pays où la gestion du patrimoine archéologique est menée en lien direct avec la recherche universitaire. Ce n'est pas le cas en France, où le ministère de la Culture, d'un côté et le CNRS ou l'Université de l'autre restent chacun dans leur pré carré. Preuve en est, l'indifférence des milieux de la recherche archéologique face aux services que pourraient rendre la carte archéologique. Certes, l'argument principal de ce désintérêt dans cette approche, pour les acteurs de l'archéologie préventive, est le manque de temps pour élaborer des modèles prédictifs (autant de modèles différents à concevoir que de grandes périodes archéologiques et dans des contextes géographiques différents). Ces modèles doivent en fait être élaborés en amont (par un projet commun entre le Ministère de la Culture et les archéologues) et mis à la disposition de l'archéologie préventive au début d'une opération. L'exemple de la Slovénie a montré l'utilité de cette approche en archéologie préventive, au moment de la construction de l'autoroute nordsud du pays dans les années 1990 (Stancic et al. 2000).

\section{Prospection intrasite et prospection à grande échelle}

Il est maintenant utile, ici, de distinguer, au niveau de la définition des méthodes et de l'emploi des techniques, le niveau d'échelle de la prospection. Ainsi, nous devons distinguer la prospection intrasite de la prospection sur une grande surface ou «à grand échelle » que j'avais aussi appelée « régionale », faute de mieux. En effet, le mot «à grande échelle», même s'il semble être passé dans la littérature notamment archéologique, est un abus de langage pour un cartographe, car son emploi à bon escient devrait être limité à définir une "carte à petite ou grand échelle », comme me l'a fait justement remarquer M. Dabas.

Dans le cas de la prospection intrasite, qui concerne aussi bien la délimitation de la superficie au sol d'un site archéologique que l'identification du site, de son urbanisme et de l'architecture de ses bâtis, les méthodes et techniques à notre disposition, permettent de résoudre la question. Je renvoie le lecteur à (Djindjian, 1991, §4.2), (Djindjian, 2001) et (Djindjian, 2011, §3) pour des développements qui sont ici en dehors du sujet traité.

En prospection programmée à grande échelle, la superficie prospectée peut varier de $10 \mathrm{~km}^{2}$ à plus de $1000 \mathrm{~km}^{2}$. Elle est le plus souvent délimitée géographiquement. En archéologie préventive, c'est la superficie de l'aménagement qui définit la surface à prospecter. Dans le cas de travaux linéaires (autoroute, lignes de TGV, canaux), la largeur sera de 300 à 500 mètres d'emprise sur une longueur pouvant atteindre plusieurs centaines de kilomètres, soit jusqu'à plus de $1000 \mathrm{~km}^{2}$. Dans le cas de sites ponctuels (usines, barrages, zones industrielles ou commerciales, zones d'habitation), la superficie sera plus proche de quelques $\mathrm{km}^{2}$. Les ordres de grandeur sont donc très différents et l'approche nécessairement aussi.

Tout d'abord, il est très important de faire remarquer une confusion dans le concept de prospection archéologique. Sous le terme de prospection, l'archéologue désigne à la fois la recherche et le repérage des sites archéologiques (mais aussi des paysages archéologiques) et ce que l'archéologie préventive française appelle la phase de diagnostic (pour laquelle est utilisée la pratique des sondages par transects à la pelle mécanique). Peut-être faudrait-il limiter 1"'emploi du mot «prospection à grande échelle » à la recherche et au repérage des sites archéologiques, par des moyens non destructifs et destructifs, que ce soit dans le cadre d'opérations de recherche programmée ou d'opérations préventives, ce qui était peu ou prou désigné autrefois par " phase d'évaluation ».

Pour le ministère de la Culture, le diagnostic archéologique est d'abord une étape administrative et semble n'avoir que secondairement un rôle scientifique et technique dans le cadre de la procédure réglementaire. Faut-il en déduire que l'archéologie française a définitivement banni la prospection à 
grande échelle et en corollaire l'archéologie prédictive depuis la loi de 2001 qui impose la procédure du diagnostic archéologique en conséquence de la saisine archéologique ?

L'utilisation de sondage par transects à la pelle mécanique, comme nous l'avons vu précédemment, n'est pas adaptée à la prospection à grande échelle pour des raisons d'efficacité. En outre, elle n'intervient que tardivement après la phase d'acquisition du foncier, et ce, malgré la déconnexion entre la propriété du foncier et la procédure réglementaire des diagnostics archéologiques. Car ces derniers sont prescrits par des arrêtés préfectoraux qui ont une valeur coercitive et un caractère d'obligation aux tiers (comme ce qui a été fait, par exemple, pour les parcelles du projet d'aéroport de Notre Dame des Landes).

La technique de sondage par transects à la pelle mécanique a son utilité sur des superficies de l'ordre de quelques $\mathrm{km}^{2}$. Mais ici également, l'application d'une prospection préalable permet de révéler des sites (même si elle ne les révèle pas tous), ce qui permettra d'améliorer l'emploi du sondage par pelle mécanique par un plan adapté plus efficace que le plan aléatoire « in terra incognita ».

Dans d'autres cas de figure, il faut faire remarquer que cette technique par sondages n'est pas la mieux adaptée. Ainsi, l'exemple du site protohistorique de Passy-sur-Yonne dans les années 1980 montre que des solutions alternatives plus efficaces peuvent être employées. Les structures archéologiques avaient été découvertes par prospection aérienne et un projet d'exploitation sablière était prévu entraînant l'intervention des archéologues. Une superficie de 1 à $2 \mathrm{~km}^{2}$ a été décapée superficiellement à la pelle mécanique révélant nettement les structures: fosses, fossés, enclos, sépultures, appartenant à l'âge du fer, à l'âge du bronze et au néolithique. Il ne restait plus à l'archéologue qu'à fouiller exhaustivement toutes les sépultures (petites structures oblongues) et échantillonner les autres (fosses, fossés, enclos, etc.). Beaucoup de sites protohistoriques peuvent être fouillés de la même manière.

Enfin, faut-il rappeler que la profondeur de localisation des sites dépend de la sédimentation quaternaire ? Les sites paléolithiques sont souvent situés à une profondeur supérieure à la longueur du bras de la pelle mécanique ou à la profondeur maximale de prescription. Le site de Biache Saint Vaast localisé à plus de dix mètres sous la surface actuelle du sol n'aurait jamais été découvert sans les travaux de fondations d'un laminoir par Usinor! Ne cherchons plus pourquoi le nombre de sites paléolithiques est-il sous-évalué dans les opérations d'archéologie préventive !

\section{Méthodologie de la prospection à grande échelle}

\subsection{Le projet}

Un projet de prospection archéologique à grande échelle implique la conception et la définition précise d'un plan de prospection, qui comporte plusieurs étapes :

1. Spécifications de l'opération

2. Documentation préalable

3. Préparation et mise en place de la logistique de terrain

4. Mise en œuvre d'un système d'information géographique

5. Elaboration et mise en œuvre du plan de prospection du territoire

- Zonages et balisages

- Choix des techniques de prospection

- Optimisation par un plan d'échantillonnage

6. Plan de prospection intrasite, sondages et fouilles des sites découverts 
7. Etudes par échantillonnage des artefacts

8. Publication du rapport de prospection

Nous ne détaillerons pas les points 1 à 4 et 6 à 8 , au profit des points critiques que sont les 5 et 6 .

\subsection{Zonages et balisages}

La région à prospecter est évidemment hétérogène tant du point de vue de la géomorphologie, de la végétation, de l'exploitation agricole que de la richesse en sites archéologiques. Il convient donc de procéder à un zonage préalable qui a pour premier objectif de structurer l'espace à prospecter, en commençant par ceux qui sont inaccessibles ou ceux dont les sites archéologiques ont été détruits pour des raisons anthropiques ou naturelles :

- Les zonages à contraintes d'accès

- Les zones de bâtis (bâtis industriels, bâtis isolés (stations), bâtis résidentiels),

- Les zones agricoles aménagées (vignes, vergers, serres, terrasses, etc.),

- Les zones naturelles exclues (zones inondées, zones montagneuses, zones érodées),

- Les zonages géomorphologiques (versants, abrupts, lits, plateaux, plaines, etc.),

- Les zonages agricoles (cultures, prairies, forêts, taillis, landes, friches, etc.),

- Les zonages artificiels pour réduire la taille des zones trop grandes,

- Macro-prospection (de 1 hectare à 100 hectares)

- Micro-prospection (moins de1 hectare)

Sur le terrain, le repérage et les balisages sont essentiels. Ceux-ci doivent être définis sur la carte du SIG et complétés quand nécessaire sur le terrain.

- Repères existants (bornes, pylônes, amers, etc.) ou ajoutés (piquets),

- Repères virtuels (triangulation, GPS).

\subsection{Le plan de prospection}

Une fois réunie la documentation préalable, paramétré le SIG et effectué un zonage et un balisage préalable, le plan de prospection doit faire le choix des techniques de prospection pour chaque zone définie à partir des techniques de prospection disponibles et énumérées ci-dessous. Une règle de bonne pratique est la suivante : "une zone = une seule unité géomorphologique + une seule technique de prospection + une seule journée de prospection avec une équipe inférieure ou égal à 4 personnes ». Mais il est recommandé si nécessaire d'appliquer à une même zone des techniques complémentaires de prospection. Cette règle simplifie l'organisation pratique de la prospection, en définissant des tâches homogènes, courtes dans le temps, plus faciles à planifier (diagramme de Gantt), à en suivre l'exécution, à en gérer les compétences requises et enfin à budgéter.

\subsubsection{La résolution du plan}

Puis, doit être réglée la question importante de la résolution dans l'acquisition (équivalent du taux d'échantillonnage d'un plan probabiliste), que ce soit du ramassage de surface, du sondage par transects ou du Lidar, ainsi que les paramètres de personnalisation liés à chaque technique utilisée. 
Ici, l'échantillonnage apparait comme une variable d'ajustement permettant d'optimiser le plan de prospection (en termes d'équilibre entre l'efficacité, le délai de réalisation et le budget en jourshommes).

\subsubsection{L'itération du plan}

Il est recommandé d'appliquer des plans successifs sur les zones où l'évidence d'une présence archéologique se pose afin de ne pas affecter inutilement des moyens importants à une zone stérile ou pauvre en artefacts. Ceci est particulièrement vrai pour les techniques faiblement mécanisées (prospection à vue, ramassage de surface, sondage par transects, carottage, etc.).

\subsubsection{Règles d'application successive}

Plusieurs techniques peuvent être utilement appliquées successivement sur une même zone selon sa richesse en vestiges archéologiques. Il est cependant nécessaire de respecter deux règles : la première est celle de la résolution croissante (cf. 5.3.2) et la seconde est celle de l'utilisation des techniques non destructives avant les techniques destructives.

\section{Techniques de prospection}

Les techniques de prospection sont très nombreuses et elles continuent à s'améliorer et à se développer. Chacune apporte des informations utiles à la détection d'un site. Certaines sont non destructives, d'autres destructives. Aucune n'est la panacée universelle. Elles se complètent, suivant la nature des terrains et des sites archéologiques :

- Existence sinon localisation précise à partir des textes de l'Antiquité,

- Emplacements topographiques privilégiés (grottes, abris sous roche, oppida, convergence de cours d'eau, terrasses de versant de vallée, etc.),

- Etudes toponymiques,

- Archives archéologiques et muséologiques,

- Prospection aérienne (récupération d'archives photographiques),

- Cheminement photographique par drone (qui remplace le cheminement pédestre),

- Etudes cartographiques (cadastre napoléonien particulièrement),

- Etudes de géomorphologie quaternaire (pour la connaissance des sédimentations et des érosions),

- Ramassage de surface (champs après labour et pluie, terrains peu ou pas sédimentés), suivant une procédure d'acquisition systématique et validée,

- Images satellites,

- Télédétection satellite (détection de réseaux),

- Lidar (en milieu forestier principalement mais pas seulement),

- Techniques de prospection géophysique (résistivité, électromagnétisme, susceptibilité magnétique, radar, magnétisme, thermographie, gravimétrie, pénétrométrie, etc.)

- Sonar en milieu sous-marin et subaquatique,

- Carottages manuels et mécaniques,

- Sondages par transects à la pelle mécanique. 


\section{Améliorations des techniques de prospection archéologique}

Depuis une petite vingtaine d'années, grâce aux progrès techniques de l'informatique et de la géophysique, une amélioration spectaculaire a été observée sur plusieurs des techniques utilisées dans la prospection archéologique :

\subsection{SIG et MNT}

Il est presque inutile de rappeler ici que les systèmes d'Information géographique sont devenus l'outil de base d'un projet de prospection archéologique. Une des conséquences de son utilisation est la facilité d'obtenir un modèle numérique de terrain (MNT), indispensable ici.

\subsection{Lidar}

Le scanner laser aéroporté ou LIDAR a été une révolution récente en archéologie par sa capacité à acquérir une microtopographie du terrain indépendamment de sa végétation grâce aux traitements informatiques associés. Cette technique a révolutionné la prospection archéologique en milieu forestier et végétal dense (Sittler et al. 2007 ; Georges-Leroy et al. 2011).

\subsection{Images satellites}

L'amélioration de la résolution des prises de vues photographiques des satellites civils, qui ont franchi le seuil de résolution du mètre, fourni aux archéologues un outil précieux de prospection. La résolution actuelle des satellites militaires qui atteint de nos jours quelques centimètres, permet d'envisager à terme une résolution de $10 \mathrm{~cm}$ pour les applications civiles (Scott Madry, 2007 ; Lecoq et Déodat, 2009).

\subsection{Usage du drone}

L'usage du drone s'est très rapidement répandu ces dernières années. En prospection, il permet d'optimiser la technique de prospection à vue dans des espaces découverts. Bien au-delà, la miniaturisation rapide des capteurs permet d'envisager le drone comme un équipement de prospection : Lidar mais aussi thermographie infrarouge ouvrant là à terme des perspectives pour la prospection géophysique aéroportée. Son temps de vol est aujourd'hui limité à une vingtaine de minutes par la faible durée de fonctionnement des batteries embarquées, ce qui implique une gestion de type « relais de poste » pour leur remplacement.

\subsection{Automatisation de la prospection géophysique}

Les techniques de la prospection géophysique en archéologie, développées depuis les années 1960, sont bien connues (Scollar et al. 1990). Leurs capacités ou incapacités à détecter telles ou telles structures archéologiques ont fait l'objet d'études approfondies depuis plus de cinquante ans. Il est donc inutile de revenir en détail sur cette question qui est connue. Par contre, la miniaturisation des équipements et l'informatisation des traitements des données d'acquisition ont facilité leur utilisation dans les années 1980. Mais surtout, la mécanisation de la prospection géophysique, en baissant les coûts à l'hectare et en réduisant considérablement le temps d'acquisition est une révolution technique et économique qui l'amène à pouvoir jouer un rôle dans la prospection à grande échelle, sur des surfaces jusqu'à 1000 ha (figure 4). 


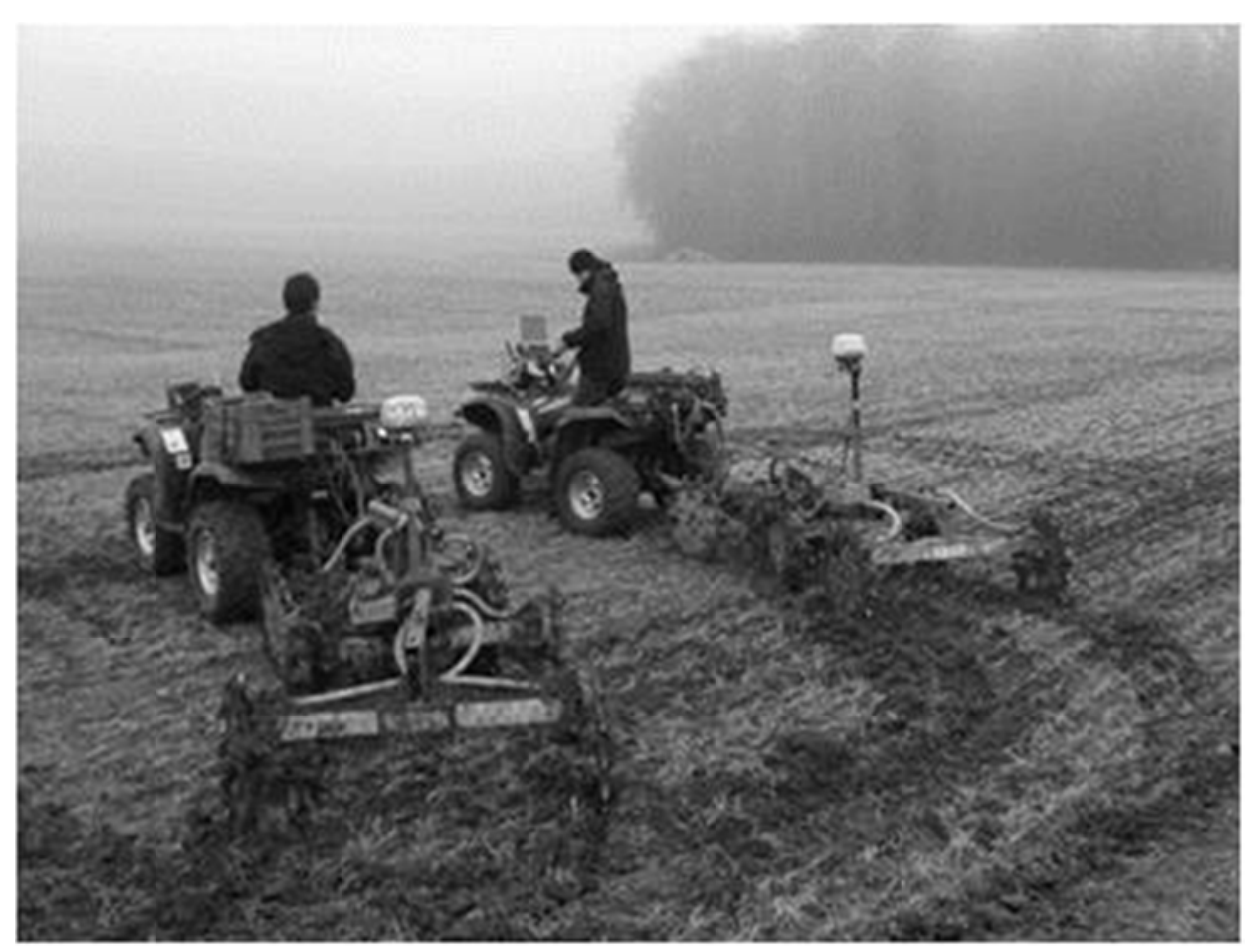

Figure 4. Machine autotractée pour la prospection géophysique

Ainsi, en quelques années, les progrès technologiques ont bouleversé notre approche technique et méthodologique de la prospection régionale à grande échelle et permettent d'envisager le recours à des techniques non destructives, dont on connait les possibilités et les limites, même si on sait qu'elles ne sont pas la solution unique pour découvrir des sites de tout type et de toute période.

\section{En France, une question politique?}

En 2014, une polémique est née de la proposition de simplification administrative $\mathrm{n}^{\circ} 14$ dans le cadre de la mission du secrétaire d'état T. Mandon : "Limiter la durée d'immobilisation des chantiers liés à l'archéologie préventive. Dans les chantiers d'archéologie préventive, les techniques alternatives de sondage seront développées, afin de "rendre les diagnostics plus rapides ». Il s'agissait notamment de développer l'usage de l'application des techniques de prospection géophysique mécanisées.

La réaction de l'INRAP, qui, en l'occurrence ici défendait son monopole des diagnostics (bien que les collectivités locales agrées puissent également les faire maintenant), fut vive et défendue par la ministre de la Culture de l'époque, A. Filippetti, dont la politique était de renforcer les missions de service public de l'INRAP, mis en concurrence par la loi du 1 août 2003 pour les opérations. Elle chargea la députée Martine Faure, d'un rapport sur l'archéologie préventive (mai 2015) et un inspecteur général du ministère, F. Fichet de Clairefontaine d'un rapport, publié en septembre 2014 : «La géophysique appliquée à la recherche archéologique en milieu terrestre, apports et limites, et confirmé par un avis du CNRA de mars 2015 sur la prospection géophysique dans les investigations archéologiques. La méthode de prospection INRAP a donc été confirmée officiellement par le ministère. La lecture de ces documents, dont le style et le vocabulaire sont caractéristiques du microcosme archéologique, montrent qu'ils ont été largement rédigés à partir des positions de l'INRAP et de ses syndicats. L'argumentation principale semble être bien résumée dans le document (Bayard D., Hulin G. Koehler A., Prilaux G., Talon M., 2015) remis au CNRA en mars 2015.

Mais c'est aussi l'éventualité que les aménageurs puissent recourir par eux-mêmes à des prospections géophysiques pour faire la démonstration au ministère de la Culture de l'absence de vestiges archéologiques qui a généré cette brutale levée de bouclier. 
Plus globalement c'est le cadre d'application du partage de responsabilités entre aménageurs (comme maîtrise d'ouvrage), ministère de la culture (comme contrôle qualité mais est-ce suffisant ?) et responsables de diagnostics et d'opérations (comme maîtrise d'œuvre) qui est concerné. Face à des améliorations techniques aussi rapides qu'inéluctables, la bonne solution est-elle de s'y opposer ou de s'y adapter intelligemment?

Cette attitude entraîne inévitablement des dégâts collatéraux dans les spin-off du CNRS spécialisés dans la prospection géophysique, qui offrent des services de qualité, avec un personnel d'anciens chercheurs qui n'auront alors d'autres solutions que de revenir dans les structures étatiques de la recherche.

Revenant sur la distinction qui a été faite précédemment entre phase de prospection à grande échelle que suit la phase de diagnostic (au sens INRAP), il semble utile d'insister sur le fait scientifique qu'il ne s'agit pas d'une mise en concurrence des deux approches mais d'une mise en œuvre successive. Il s'agit ensuite d'une décision politique de décider si ces deux phases doivent être menées dans le cadre du monopole de l'INRAP faisant appel à des sous-traitants spécialisés ou suivant un mode ouvert. Il semble à l'auteur de ces lignes qu'il y ait eu, volontairement ou non, une confusion entre les deux approches.

Car c'est aussi le rôle du ministère de la Culture de définir les bonnes pratiques, c'est-à-dire les méthodes pour élaborer des plans de prospection et les modèles prédictifs à partir des données de la carte archéologique, qui doivent être employés dans les relations entre maitre d'ouvrage et maitre d'œuvre de l'opération préventive. La phase de détection et la phase de diagnostics pourront être alors confiées à l'INRAP à la condition que tout maître d'œuvre fasse appel à des consultants pour élaborer les plans de prospection et à des sous-traitants pour des prospections «ad hoc » dans le cadre de marchés publics. En réduisant l'imprécision des diagnostiques, une telle démarche permettra aussi une réduction des contentieux, avec les opérateurs privés comme avec les aménageurs.

\section{L'enseignement de la prospection archéologique}

Une objection qui pourrait être faite à l'approche à valeur ajoutée proposée précédemment, mais que mettent en œuvre les chercheurs (Campana et al. 2009; Campana et al. 2011) dans des projets internationaux, est la multiplicité des compétences requises pour la mettre en œuvre et à la difficulté de les intégrer. Ce n'est à notre avis qu'une question de formation. Ainsi à l'Université de Paris 1 Panthéon Sorbonne, le cours de Master 2 de Systèmes d'Information Géographique a fait réaliser par les étudiants un projet de plan de prospection archéologique à grande échelle pendant dix ans de 2005 à 2015.

Le cahier des charges du Projet de prospection archéologique était le suivant :

- Créer une carte avec le logiciel Arcview,

- La géo-référencer,

- Lire et interpréter la carte,

- Effectuer un zonage préliminaire,

- Baliser le zonage à partir d'éléments cartographiques existants (bornes, poteaux, arbres, pylônes électriques, etc.), rajoutés (piquets) ou virtuels (coordonnées GPS),

- Réitérer de façon à obtenir un zonage définitif,

- Faire un numérotage des zones et calculer leur surface, 
- Choisir une technique de prospection appropriée pour chaque zone: prospection aérienne, prospection géophysique, ramassage de surfaces, carottages, sondages manuels $(4 \mathrm{~m} \times 4 \mathrm{~m})$, sondages par engins (transectss de $20 \mathrm{~m} \times 2 \mathrm{~m} \times 2 \mathrm{~m}$ ), Lidar, etc.

- Définir un échantillonnage pour les sondages et les carottages en appliquant un plan systématique non aligné avec un taux de 12,5\% ou inférieur,

- Évaluer la charge de prospection pour chaque zone en utilisant le référentiel de charge unitaire,

- Réaliser un planning (Gantt) et un budget en J/H de l'opération de prospection.

Il faut insister sur la capacité des étudiants à avoir rendu de bons projets, année après année ! Il faut donc noter aussi leur déception quand, lors d'un stage, on leur fait comprendre que le seul travail à faire consistait à suivre la progression d'une pelle mécanique !

\section{Conclusions}

Il est nécessaire de distinguer deux phases de prospection, la prospection archéologique à grande échelle qui a recours à un panel diversifié de techniques de prospection intégrées dans un plan de prospection optimisé, appliqué très en amont dans le cadre d'une archéologie préventive, et le diagnostic, qui intervient ensuite et dont le plan est optimisé par les résultats de la phase précédente. Les deux phases ne sont pas concurrentes mais complémentaires. Le budget actuel assuré par la redevance d'archéologie préventive doit permettre de prendre ces coûts en charge et ce d'autant plus facilement que le montant des marchés de prestations techniques de l'INRAP (engins de chantier) sera d'autant abaissé que les dépenses seront redirigées vers les méthodes non destructives. Elle offre en outre une sécurité supplémentaire avec une gestion du calendrier beaucoup plus en amont, bien avant l'acquisition du foncier.

La validation des méthodes de prospection reste cependant le meilleur juge de paix en la matière. Ainsi il aurait été utile de connaitre le retour d'expérience de l'INRAP sur près de 20 ans d'utilisation systématique de la prospection par sondage par transects à la pelle mécanique. Il suffit en effet de croiser les données des diagnostics archéologiques, les prescriptions de fouilles et le résultat de ces dernières, projet d'étude que le ministère de la Culture pourrait utilement mettre en chantier. De même, il serait utile d'étudier les retours d'expérience de nos voisins européens et de s'inspirer de ce qu'ils font mieux que nous.

\section{Bibliographie}

BAYARD D., HULIN G. KOEHLER A., PRILAUX G., TALON M. (2015) : prospections géophysiques et diagnostic archéologique: l'expérience du canal Seine-Nord Europe. Conseil national de Centre National de la recherche archéologique, Ministère de la Culture, séance du 26 mars2015

BLOUET V., 1994, Essais de comparaison de différentes méthodes d'étude archéologiques préalables, Les Nouvelles de l’Archéologie, 1994, 58, p.17-19).

CAMPANA S., PIRO S. (ed.) (2009) : Seeing the Unseen. London, Taylor \& Francis

CAMPANA S., DABAS M., (2011) : Archaeological Impact Assessment: The Brebemi Project (Italy), in Archaeological Prospection, Special Issue on "Geophysical Investigations for Archaeological Prospection”, 18, 2, p.139-148.

CARTIER CL., HESSE A., CARTIER J., CHAPELOT J., CHAPELOT O., TABBAGH A. (1972) : L'artisanat céramique en Saintonge (XIIIe siècle-XIXe siècles) : essai d'archéologie extensive, terrestre et subaquatique : rapport préliminaire. Paris, Musée National des arts et traditions populaires ; Ecole pratique des Hautes Etudes VIème section, $108 \mathrm{p}$.

CHERRY F., GAMBLE C., SHENNAN S. eds. (1978) : Sampling in contemporary British archaeology, Oxford, Archaeopress (British Archaeological Reports, 50) 
DABAS M., DELETANG H., FERDIERE A., JUNG C., ZIMMERMANN H. (1998) : La prospection, Paris, Editions Errance (Collection Archéologiques)

DESABIE J. (1966) : Théorie et pratique des sondages, Paris, Dunod (Statistique et programmes économiques ; 10)

DJINDJIAN, F. (1991) : Méthodes pour l'Archéologie. Paris, Armand Colin, 1991.

DJINDJIAN F. (2001) : Prospection archéologique au sol, de la région au site, in « Géologie de la Préhistoire, J.CL. Miskovsky ed. », Paris, Geopre, Nouvelle Edition

DJINDJIAN F (2011) : Manuel d'Archéologie. Paris, Armand Colin

FERDIERE A., ZADORA-RIO E. (dir.) (1986) : La prospection archéologique- Paysage et peuplement (Actes de la table ronde de Paris 14-15 mai 1982. D.A.F., 3, Paris, MSH, 178p.

FERDIERE A., RIALLAND Y. (1995) : La prospection archéologique systématique sur le tracé de l'autoroute A 71 (section Bourges-sud du Cher). 2e partie. Revue archéologique du Centre de la France, 1995, 34, 1, p.5-87

FICHET DE CLAIREFONTAINE F. (2014) : «La géophysique appliquée à la recherche archéologique en milieu terrestre, apports et limites », n²014-31, Ministère de la Culture,

GARDIN J.-C. (1998): Prospections archéologiques en Bactriane orientale (1974-1978), sous la direction de J.-C. Gardin, vol. 3, Description des sites et notes de synthèse, MMAFAC IX, ERC, Paris.

GEORGES-LEROY M., BOCK J., DAMBRINE E., DUPOUEY J.L., LAFFITE J.D. (2014) : Parcellaires et habitat antiques des forêts du plateau de Haye en Lorraine : bilan et perspectives. Les parcellaires conservés sous forêt, Paris, France.

HESSE A. (1994) : La prospection archéologique : des mesures extensives sur deux dimensions de l'espace. Histoire et Mesure, IX, 3-4, p.213-229

LECOQ P., DEODAT L. (2009): Images satellitaires et prospection archéologique : un cas d'école dans les Andes péruviennes, Les Nouvelles de l'Archéologie, 117, p.57-61

LEUSEN M. VAN, KAMERMANS H.eds (2005) : Predictive Modeling for Archaeological Heritage Management: A research agenda, Amersfoort, ROB, (Nederlandse Archeologische Rapporten, 29)

LONDON J. (2009) : Romans, récits et Nouvelles du Grand Nord, collection Bouquins, Paris, Robert Laffont

MADRY S. (2007) : An Evaluation of Google Earth for Archaeological Aerial Prospection and Site Survey, in: Digital Discovery: Exploring New Frontiers in Human Heritage. CAA 2006, Computer Applications and Quantitative Methods in Archaeology, Proceedings of the 34th Conference, Fargo, United States, April 2006, Jeffrey T. Clark and Emily M. Hagemeister Editors. 2007. Budapest: Archaeolingua. (ISBN 978-963-8046-90-1)

MARMET M., BEST C., TABBAGH A. (2002) : Prospection systématique par sondages à la pelle mécanique : limites liées à la probabilité de découverte de sites archéologiques.Revue d'Archéométrie, 26, p.11-21

MATHERON G. (1971) : La Théorie des variables régionalisées et ses applications,Paris, École Nationale Supérieure des Mines, (Les Cahiers du Centre de morphologie mathématique de Fontainebleau ; 5)

MATHERON G. (1962): Traité de géostatistique appliquée, tome I. In E. Technip (ed.), Mémoires du Bureau de recherches géologiques et minières, $\mathrm{n}$ 14. Paris

MUELLER J. W. ed (1975) : Sampling in archaeology, Tucson, The University of Arizona Press

ROBERT S. (2003) : L'analyse morphologique entre archéologie, urbanisme et aménagement du territoire. Exemples d'études de formes urbaines et rurales dans le Val-d'Oise. Histoire, Université Panthéon-Sorbonne - Paris I, 2003, <tel$00371063>$

SCOLlAR I., TABBAGH A., HESSE A., HERZOG I. (1990) : Archaeological Prospecting and Remote Sensing, Cambridge, Cambridge University Press, 674p.

SITTLER B., WEINACKER H., GÜTLINGER M., KOUMALIANTZ L. (2007) : The potential of Lidar assessing elements of cultural hidden under forests. In "Bochenek Z. ed., New developments in Remote sensing". Millpress, Rotterdam, p.539-548

STANCIC, Z., VELJANOVSKI, T. (2000) : Understanding Roman settlement patterns through multivariate statistics and predictive modelling, in «Lock, G. ed., Beyond the map -Archaeology and Spatial Technologies », NATO Science Series, IOS Press, p.147-157. 
VESCELIUS G. S. (1960) : Archaeological sampling: a problem of statistical inference, in "Essays in the science of culture in honor of L. A. White / ed. G. A. Dole, R. L. Cameiro", New York, Crowell Co, p.457-470

WESTCOTT K.L., BRANDON J. ed. (2002) :Practical Applications of GIS for Archaeologists, London, Taylor \& Francis, 160 p., CD-ROM

\section{Remerciements}

Je tiens à remercier tout particulièrement Lionel Orengo, Michel Dabas, Alain Tabbagh et Laurent Costa pour les remarques et corrections qui ont permises d'améliorer significativement ce texte. 